\title{
Homology Groups and Foldings on Buchdahi Space
}

\author{
A. E. El-Ahmady ${ }^{1 *}$ and M. Abu-Saleem ${ }^{2}$ \\ 'Mathematics Department, Faculty of Science, Taibah University, Madinah, Saudi Arabia; \\ 'Mathematics Department, Faculty of Science, Tanta University, Tanta, Egypt; a_elahmady@hotmail.com \\ 2Department of Mathematics, Allaith University College (Girls Branch), Umm Al-Qura University, \\ Saudi Arabia; mohammedabusaleem2005@yahoo.com
}

\begin{abstract}
The purpose of this paper is to give a combinatorial characterization and also construct representations of the homology group of the submanifolds of Buchdahi space by using some geometrical transformations. The homology groups of the limit Buchdahi space are presented. The homology groups of some types of geodesics in Buchdahi space are discussed. Theorems governing this connection are achieved.
\end{abstract}

Keywords: Buchdahi Space, Homology Group, Folding, Retractions, Deformation Retract.

Mathematics Subject Classification: 53A35, 51H20, 57N10.

\section{Introduction and Definitions}

Buchdahi space represents one of the most intriguing and emblematic discoveries in the history of geometry. Although if it were introduced for a purely geometrical purpose, they came into prominence in many branches of mathematics and physics. This association with applied science and geometry generated synergistic effect: applied science gave relevance to Buchdahi space and Buchdahi space allowed formalizing practical problems $[6,8,21]$.

In vector spaces and linear maps; topological spaces and continuous maps; groups and homomorphisms together with the distinguished family of maps is referred to as a category. An operator which assigns to every object in one category a corresponding object in another category and to every map in the first a map in the second in such a way that compositions are preserved and the identity map is taken to the identity map is called a functor. Thus, we may summarize our activities thus far by saying that we have constructed a functor (the fundamental group functor) from the category of pointed spaces and maps to the category of groups and homomorphisms. Such functors are the vehicles by which one translates topological problems into algebraic problem $[10,12,14,29,35-38]$.

Most folding problems are attractive from a pure mathematical standpoint, for the beauty of the problems themselves. The folding problems have close connections to important industrial applications. Linkage folding has applications in robotics and hydraulic tube bending. Paper folding has application in sheet-metal bending, packaging, and air -bag folding [11]. Also, used folding to solve difficult problems related to shell structures in civil engineering and aero space design, namely buckling instability [11]. Isometric folding between two Riemannian manifold may be characterized as maps that send piecewise geodesic segments to a piecewise geodesic segments of the same length [7]. For a topological folding the maps do not preserves lengths [19], i.e. A map $\mathfrak{I}: M \rightarrow N$, where $\mathrm{M}$ and $\mathrm{N}$ are $c^{\infty}$-Riemannian manifolds of dimension $\mathrm{m}$ and $\mathrm{n}$ respectively is said to be an isometric folding of $\mathrm{M}$ into $\mathrm{N}$, iff for any piecewise geodesic path $\gamma: J \rightarrow M$, the induced path I $\gamma: J \rightarrow N$ is a piecewise geodesic and of the same length as $\gamma$. If $\mathfrak{I}$ does not preserve length, then $\mathfrak{I}$ is a topological folding $[15,17,32,34]$.

* Corresponding author:

A. E. El-Ahmady (a_elahmady@hotmail.com) 
An n-dimensional topological manifold $\mathrm{M}$ is a Hausdorff topological space with a countable basis for the topology which is locally homeomorphic to $R^{n}$. If $\mathrm{h}: U \rightarrow U$ ' is a homeomorphism of $U \subseteq M$ onto $U^{\prime} \subseteq R^{n}$, then $\mathrm{h}$ is called a chart of $\mathrm{M}$ and $\mathrm{U}$ is the associated chart domain. A collection $\left(h_{\alpha}, u_{\alpha}\right)$ is said to be an atlas for $\mathrm{M}$ if $\bigcup_{\alpha \in A} U_{\alpha}=M$. Given two charts $h_{\alpha}, h_{\beta}$ such that $U_{\alpha \beta}=U_{\alpha} \cap U_{\beta} \neq \varnothing$, the transformation chart $h_{\beta} \circ h_{\alpha}^{-1}$ between open sets of $R^{n}$ is defined, and if all of these charts transformation are $c^{\infty}$-mappings, then the manifolds under consideration is a $c^{\infty}$ - manifolds. A differentiable structure on $\mathrm{M}$ is a differentiable atlas and a differentiable manifold is a topological manifold with a differentiable structure [40-42]. M may have other structures as colors, density or any physical structures. The number of structures may be infinite. In this case the manifold is said to be a chaotic manifold and may become relevant to vacuum fluctuation and chaotic quantum field theories. The magnetic field of a magnet bar is a kind of chaotic 1-dimensional manifold represented by the magnetic flux lines. The geometric manifold is the magnetic bar itself [1] .

Fuzzy manifolds are special type of the category of chaotic manifolds [2-5]. Usually we denote by $\mathrm{M}=M_{012 \ldots h}$ to a chaotic manifolds, where $M_{0 h}$ is the geometric (essential) manifold and the associated pure chaotic manifolds, the manifolds with physical characters, are denoted by $M_{1 h}, \ldots, M_{\infty h}[16,24,26,27,29,30]$.

A subset $A$ of a topological space $X$ is called a retract of $X$ if there exists a continuous map $r: X \rightarrow A$ such that $r(a)=a, \forall a \in A$ where $A$ is closed and $X$ is open [9, 13, 20,31]. Also, let $X$ be a space and $A$ a subspace. A map $r: X \rightarrow A$ such that $r(a)=a$, for all $a \in A$, is called a retraction of $X$ onto $A$ and $A$ is the called a retract of $X$. This can be re stated as follows. If $i: X \rightarrow A$ is the inclusion map, then $r: X \rightarrow A$ is a map such that $r i=i d_{a}$. If, in addition, $r i \approx i d_{X}$, we call $r$ a deformation retract and $A$ a deformation retract of $X$. Another simple-but extremely useful-idea is that of a retract. If $A, X \subset M$, then $A$ is a retract of $X$ if there is a commutative diagram $[18,22,23,28]$.

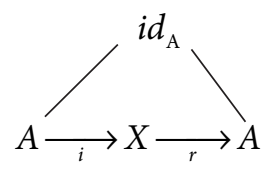

Homology theory is the axiomatic study of the intuitive geometric idea of homology of cycles on topological spaces, to any topological space $X$ and any natural number $n$, one can associate a set $H_{n}(X)$, whose elements are called (n-dimensional) homology classes. There is a well-defined way to add and subtract homology classes, which makes $H_{n}(X)$ into an abelian group, called the $\mathrm{n}$-th homology group of $X$. In heuristic terms, the size and structure of $H_{n}(X)$ gives information about the number of $\mathrm{n}$-dimensional holes in $X$. For example, if $X$ is a figure eight, then it has two holes, which in this context count as being one-dimensional. The corresponding homology group $H_{1}(X)$ can be identified with the group $Z \otimes Z$ of pairs of integers, with one copy of $Z$ for each hole. While it seems very straightforward to say that $X$ has two holes, it is surprisingly hard to formulate this in a mathematically rigorous way; this is a central purpose of homology theory $[25,40]$.

A singular $\mathrm{n}$-simplex is a continuous mapping $\sigma_{n}$ from the standard n-simple $\Delta^{n}$ to a topological space $X$. Notationally, one writes $\sigma_{n}: \Delta^{n} \rightarrow X$. This mapping need not be injective, and there can be non-equivalent singular simplexes with the same image in $X$.

The boundary of $\sigma_{n}\left(\Delta^{n}\right)$, denoted as $\partial_{n}\left(\sigma_{n}\left(\Delta^{n}\right)\right)$, is defined to be the formal sum of the singular $(n-1)$-simplexes represented by the restriction of $\sigma$ to the faces of the standard $n$-simplex, with an alternating sign to take orientation into account. A formal sum is an element of the free abelian group on the simplexes. The basis for the group is the infinite set of all possible images of standard simplexes. The group operation is "addition" and the sum of image $a$ with image $b$ is usually simply designated $a+b$, but $a+a=2 a$ and so on. Every image $a$ a has a negative $-a$. Thus, if we designate the range of $\sigma_{n}$ by its. Vertices $\left[p_{0}, p_{1}, \ldots, p_{n}\right]=\left[\sigma_{n}\left(e_{0}\right), \sigma_{n}\left(e_{1}\right), \ldots, \sigma_{n}\left(e_{n}\right)\right]$ Corresponding to the vertices $e_{k}$ of the standard $n$-simplex $\Delta^{n}$ (which of course does not fully specify the standard simplex image produced by $\sigma_{n}$, and then $\partial_{n}\left(\sigma_{n}\left(\Delta^{n}\right)\right)=\sum_{k=0}^{n}(-1)^{n}\left[p_{0}\right.$ $\left.p_{1}, \ldots, p_{k-1}, p_{k+1}, \ldots, p_{n}\right]$ is a formal sum of the faces of the simplex image designated in a specific way. That is, a particular face has to be the image of $\sigma_{n}$ applied to a designation of a face of $\Delta^{n}$ which depends on the order that its vertices are listed.) Thus, for example, the boundary of $\sigma\left(\left[p_{0}, p_{1}\right]\right)$ (acurve going from $p_{0}$ to $p_{1}$ ) is the formal sum or "formal difference" $\left[p_{1}\right]-\left[p_{0}\right]$.

Singular chain complex, the usual construction of singular homology proceeds by defining formal sums of simplexes, which may be understood to be elements of a free abelian group, and then showing that we can define a certain group, the homology group of the topological space, involving the boundary operator [27].

Consider first the set of all possible singular n-simplexes $\sigma_{n}\left(\Delta^{n}\right)$ on a topological space $X$. This set may be used as the basis of a free abelian group, so that each $\sigma_{n}\left(\Delta^{n}\right)$ is a generator of the group. This set of generators is of course usually infinite, frequently uncountable, as there are many 
ways of mapping a simplex into a typical topological space. The free abelian group generated by this basis is commonly denoted as $C_{n}(X)$. Elements of $C_{n}(X)$ are called singular $\mathrm{n}$-chains; they are formal sums of singular simplices with integer coefficients. In order for the theory to be placed on a firm foundation, it is commonly required that a chain be a sum of only a finite number of simplexes. The boundary $\partial$ is readily extended to act on singular n-chains. The extension, called the boundary operator, written as $\partial_{n}: C_{n} \rightarrow C_{n-1}$ is a homomorphism of groups. The boundary operator, together with the $C_{n}$ form a chain complex of abelian groups, called the singular complex. It is often denoted as $\left(C .(X), \partial_{\bullet}\right)$ or more simply $C_{\mathbf{.}}(X)[33,35]$. The kernel of the boundary operator is $Z_{n}(X)=\operatorname{ker}\left(\partial_{n}\right)$, and is called the group of singular $n$-cycles. The image of the boundary operator is $B_{n}(X)=i m\left(\partial_{n+1}\right)$, and is called the group of singular $n$-boundaries. It can also be shown that $\partial_{n} \partial_{n+1}=0$. The $n$-th homology group of $X$ is then defined as the factor group $H_{n}(X)=Z_{n}(X) / B_{n}(X)$. The elements of $H_{n}(X)$ are called homology classes $[33,39]$.

\section{Main Results}

Theorem 1: The first homology group on types of the deformation retracts of Buchdahi space $\beta^{4}$ is either identity group or isomorphic to $Z \oplus Z$.

Proof: Consider the Buchdahi space $\beta^{4}$. Used cylindrical coordinates $z, r, \theta$ and $t$, with metric

$$
d s^{2}=-\gamma^{2}\left(\gamma^{-1} d r^{2}+r^{2} d \theta^{2}+r^{2} \sin ^{2} \theta d \phi^{2}+p^{-1} d t^{2}\right)
$$

The coordinates of Buchdahi space $\beta^{4}$ are given by

$$
\left.\begin{array}{l}
x_{1}=\frac{A_{1}}{1-i \sqrt{\gamma}} \sin \left(\frac{A_{2}}{1-i r \gamma}\right) \cos \left(\frac{A_{3}}{1-i r \gamma \sin \phi}\right) \\
x_{2}=\frac{A_{1}}{1-i \sqrt{\gamma}} \sin \left(\frac{A_{2}}{1-i r \gamma}\right) \sin \left(\frac{A_{3}}{1-i r \gamma \sin \phi}\right) \\
x_{3}=\frac{A_{1}}{1-i \sqrt{\gamma}} \cos \left(\frac{A_{2}}{1-i r \gamma}\right) \\
x_{4}=\frac{A_{4}}{1-\sqrt{p-1}}
\end{array}\right\}
$$

where $A_{1}, A_{2}, A_{3}$, and $A_{4}$ are the constant of integration, Now, we use Lagrangian equations $\frac{d}{d s}\left(\frac{\partial T}{\partial G_{i}}\right)-$ $\frac{\partial T}{\partial G_{i}}=0, i=1,2,3,4$. To find a geodesic which is a subset of the Buchdahi space $\beta^{4}$. Since

$$
T=\frac{1}{2}\left\{-\gamma^{2}\left(\gamma^{-1} r^{2}+r^{2} \theta^{\prime 2}+r^{2} \sin ^{2} \theta \phi^{2}+p^{-2} t^{2}\right\}\right.
$$

Then the Lagrangian equations for Buchdahi space $\beta^{4}$ are

$$
\begin{aligned}
& \frac{d}{d s}\left(\gamma r^{\prime}\right)+\left(-\gamma^{2} r \theta^{\prime 2}-\gamma^{2} r \sin ^{2} \theta \phi^{\prime 2}\right)=0 \\
& \frac{d}{d s}\left(\gamma^{2} r^{2} \theta\right)+\left(\gamma^{2} r^{2} \sin \theta \cos \theta \phi^{\prime 2}\right)=0 \\
& \frac{d}{d s}\left(\gamma^{2} r^{2} \sin ^{2} \theta \phi^{\prime}\right)=0 \\
& \frac{d}{d s}\left(p^{-1} t^{\prime}\right)=0
\end{aligned}
$$

From equation (5) we obtain $\gamma^{2} r^{2} \sin ^{2} \theta \phi^{\prime}=$ constant say $\beta_{1}$, if $\beta_{1}=0$, we obtain the following cases. If $\gamma^{2}=0$, then the coordinates of Buchdahi space $\beta^{4}$ are given by $\left(A_{1} \sin A_{2}\right.$ $\left.\cos A_{3}, A_{1} \sin A_{2} \sin A_{3}, A_{1} \cos A_{2}, \frac{A_{4}}{1-\sqrt{p-1}}\right)$, which is hypersphere $S_{1}^{3} \subset \beta^{4}$ which is a geodesic retraction. If $r^{2}=0$, hence we get the hypersphere $S_{1}^{0} \subset \beta^{4}$ on the null cone which is a geodesic retraction. If $\phi^{\prime}=0$, then $\phi=$ constant say $\beta_{2}$, if $\beta_{2}$, then we obtain the following geodesic retraction $S_{2}^{3} \subset \beta^{4}$. The deformation retracts of the Buchdahi space $\beta^{4}$ is defined by $\eta:\left\{\beta^{4}-\left(\delta_{i}\right)\right\} \times I \rightarrow\left\{\beta^{4}-\left(\delta_{i}\right)\right\}$, where $\left\{\beta^{4}-\left(\delta_{i}\right)\right\}$ is the open Buchdahi space $\beta^{4}$ and $I$ is the closed interval $[0,1]$. The retractions of the open Buchdahi space $\beta^{4}$ is $R:\left\{\beta^{4}-\left(\delta_{i}\right)\right\} \rightarrow S_{1}^{3}, S_{1}^{0}, S_{2}^{3}$.

The deformation retract of $S_{1}^{3} \subset \beta^{4}$ is given by

$$
\begin{aligned}
& \eta(m, c)=\cos \frac{\pi c}{2}\left\{\frac{A_{1}}{1-i \sqrt{\gamma}} \sin \left(\frac{A_{2}}{1-i r \gamma}\right) \cos \left(\frac{A_{3}}{1-i r \gamma \sin (\varphi)}\right),\right. \\
& \frac{A_{1}}{1-i \sqrt{\gamma}} \sin \left(\frac{A_{2}}{1-i r \gamma}\right) \sin \left(\frac{A_{3}}{1-i r \gamma \sin (\varphi)}\right), \frac{A_{1}}{1-i \sqrt{\gamma}} \cos \left(\frac{A_{2}}{1-i r \gamma}\right), \\
& \left.\frac{A_{4}}{1-\sqrt{p-1}}-\left(\delta_{i}\right)\right\}+\sin \frac{\pi c}{2}\left\{A_{1} \sin A_{2} \cos A_{3}, A_{1} \sin A_{2}\right. \\
& \left.\sin A_{3}, A_{1} \cos A_{2}, \frac{A_{4}}{1-\sqrt{p-1}}\right\},
\end{aligned}
$$

where

$$
\begin{aligned}
& \eta(m, 0)=\left\{\frac{A_{1}}{1-i \sqrt{\gamma}} \sin \left(\frac{A_{2}}{1-i r \gamma}\right) \cos \left(\frac{A_{3}}{1-i r \gamma \sin (\varphi)}\right), \frac{A_{1}}{1-i \sqrt{\gamma}} \sin \left(\frac{A_{2}}{1-i r \gamma}\right)\right. \\
& \left.\sin \left(\frac{A_{3}}{1-i r \gamma \sin (\varphi)}\right), \frac{A_{1}}{1-i \sqrt{\gamma}} \cos \left(\frac{A_{2}}{1-i r \gamma}\right), \frac{A_{4}}{1-\sqrt{p-1}}-(\delta)\right\}, \text { and } \\
& \eta(m, 1)=\left\{A_{1} \sin A_{2} \cos A_{3}, A_{1} \sin A_{2} \sin A_{3}, A_{1} \cos A_{2}, \frac{A_{4}}{1-\sqrt{p-1}}\right\}
\end{aligned}
$$

The deformation retract of $S_{1}^{0} \subset \beta^{4}$ is defined as

$$
\begin{gathered}
\eta(m, c)=\frac{1-c}{1+c}\left\{\frac{A_{1}}{1-i \sqrt{\gamma}} \sin \left(\frac{A_{2}}{1-i r \gamma}\right) \cos \left(\frac{A_{3}}{1-i r \gamma \sin (\varphi)}\right),\right. \\
\frac{A_{1}}{1-i \sqrt{\gamma}} \sin \left(\frac{A_{2}}{1-i r \gamma}\right) \sin \left(\frac{A_{3}}{1-i r \gamma \sin (\varphi)}\right), \frac{A_{1}}{1-i \sqrt{\gamma}} \cos \left(\frac{A_{2}}{1-i r \gamma}\right), \\
\left.\frac{A_{4}}{1-\sqrt{p-1}}-\left(\delta_{i}\right)\right\}+c(2 c-1)\{0,0,0,0\} .
\end{gathered}
$$


The deformation retract of $S_{2}^{3} \subset \beta^{4}$ is defined as

$$
\begin{aligned}
& \eta(m, c)=\ln e^{(1-c)}\left\{\frac{A_{1}}{1-i \sqrt{\gamma}} \sin \left(\frac{A_{2}}{1-i r \gamma}\right) \cos \left(\frac{A_{3}}{1-i r \gamma \sin (\varphi)}\right), \frac{A_{1}}{1-i \sqrt{\gamma}}\right. \\
& \sin \left(\frac{A_{2}}{1-i r \gamma}\right) \sin \left(\frac{A_{3}}{1-i r \gamma \sin (\varphi)}\right), \frac{A_{1}}{1-i \sqrt{\gamma}} \cos \left(\frac{A_{2}}{1-i r \gamma}\right), \\
& \left.\frac{A_{4}}{1-\sqrt{p-1}}-\left(\delta_{i}\right)\right\}+\ln e^{c}\left\{\frac{A_{1}}{1-i \sqrt{\gamma}} \sin \left(\frac{A_{2}}{1-i r \gamma}\right), 0, \frac{A_{1}}{1-i \sqrt{\gamma}} \cos \left(\frac{A_{2}}{1-i r \gamma}\right),\right. \\
& \left.\frac{A_{4}}{1-\sqrt{p-1}}\right\} .
\end{aligned}
$$

Thus $H_{1}\left\{\beta^{4}-\left(\delta_{i}\right)\right\} \approx H_{1}\left(S_{1}^{3}\right), \quad H_{1}\left\{\beta^{4}-\left(\delta_{i}\right)\right\} \approx H_{1}\left(S_{1}^{0}\right)$ and $H_{1}\left\{\beta^{4}-\left(\delta_{i}\right)\right\} \approx H_{1}\left(S_{2}^{3}\right)$. Therefore $H_{1}\left(S_{1}^{3}\right), H_{1}\left(S_{1}^{0}\right)$ and $H_{1}\left(S_{2}^{3}\right)$ are either isomorphic to identity group or $Z \oplus Z$.

Corollary 1: The first homology group of types of the deformation retracts of $S_{1}^{3} \subset\left\{\beta^{4}-\left(\delta_{i}\right)\right\}$ and any manifold homeomorphic Buchdahi space $\left\{\beta^{4}-\left(\delta_{i}\right)\right\}$ is either identity group or isomorphic to $Z \oplus Z$.

Theorem 2: The homology group of the limit of foldings of the hypersphere $S_{1}^{3} \subset\left\{\beta^{4}-\left(\delta_{i}\right)\right\}$ and any manifold homeomorphic to it is either identity group or isomorphic to $Z$.

Proof: Consider the hypersphere $S_{1}^{3}$ and let $\eta_{1}: S_{1}^{3} \rightarrow S_{1}^{3}$ be a folding map, now we can define a series of folding maps by $\eta_{2}: \eta_{1}\left(S_{1}^{3}\right) \rightarrow \eta_{1}\left(S_{1}^{3}\right), \quad \eta_{3}: \eta_{2}\left(\eta_{1}\left(S_{1}^{3}\right)\right) \rightarrow \eta_{2}\left(\eta_{1}\left(S_{1}^{3}\right)\right), \ldots$, $\eta_{n}: \eta_{n-1}\left(\eta_{n-2} \ldots\left(\eta_{1}\left(S_{1}^{3}\right)\right) \ldots\right) \rightarrow \eta_{n-1}\left(\eta_{n-2} \ldots\left(\eta_{1}\left(S_{1}^{3}\right)\right) \ldots\right)$, then $\lim _{n \rightarrow \infty}\left(\eta_{n}\left(\eta_{n-1}\left(\eta_{n-2} \ldots\left(\eta_{1}\left(S_{1}^{3}\right)\right) \ldots\right)\right)=S_{1}^{2}\right.$, is a great sphere of dimension two. Therefore, $H_{n}\left(S_{1}^{2}\right)$ is isomorphic to identity group for $n \neq 0, n \neq 2$ and $H_{n}\left(S_{1}^{2}\right) \approx Z$ for $n=0$, 2. Also, if we consider $\gamma_{1}: S_{1}^{2} \rightarrow S_{1}^{2}$, $\gamma_{2}: \gamma_{1}\left(S_{1}^{2}\right) \rightarrow \gamma_{1}\left(S_{1}^{2}\right), \quad \gamma_{3}: \gamma_{2}\left(\gamma_{1}\left(S_{1}^{2}\right)\right) \rightarrow \gamma_{2}\left(\gamma_{1}\left(S_{1}^{2}\right)\right), \ldots$, $\gamma_{m}: \gamma_{m-1}\left(\gamma_{m-2} \ldots\left(\gamma_{1}\left(S_{1}^{2}\right)\right) \ldots\right) \rightarrow \gamma_{m-1}\left(\gamma_{m-2} \ldots\left(\gamma_{1}\left(S_{1}^{2}\right)\right) \ldots\right)$, then $\lim _{m \rightarrow \infty} \gamma_{m}\left(\gamma_{m-1}\left(\gamma_{m-2} \ldots\left(\gamma_{1}\left(S_{1}^{2}\right)\right) \ldots\right)=s_{1}^{1} \subset \beta^{4}\right.$, which is the great circle of dimension one. Therefore $H_{n}\left(S_{1}^{1}\right) \approx Z$ is isomorphic to $Z$ for $n=0,1$ and $H_{n}\left(S_{1}^{1}\right)=0$ for $n \geq 2$.

Theorem 3: Under the folding $\Pi_{m}\left(x_{1}, x_{2}, x_{3}, x_{4}\right)=\left(x_{1}, x_{2}\right.$, $\left.x_{3},\left|\frac{x_{4}}{m}\right|\right)$, the homology group of the limit of foldings and any manifold homeomorphic to this type of folding of $S_{2}^{3}$ is isomorphic to $Z$.

Proof: Consider the geodesic retraction hypersphere $S_{2}^{3}$ and let $\prod_{m}: S_{2}^{3} \rightarrow S_{2}^{3}$ be given by $\Pi_{m}\left(x_{1}, x_{2}, x_{3}, x_{4}\right)=$ $\left(x_{1}, x_{2}, x_{3},\left|\frac{x_{4}}{m}\right|\right)$. Then, the isometric chain folding of $S_{2}^{3}$ into itself defined by

$$
\Pi_{1}:\left\{\frac{A_{1}}{1-i \sqrt{\gamma}} \sin \left(\frac{A_{2}}{1-i r \gamma}\right), 0, \frac{A_{1}}{1-i \sqrt{\gamma}} \cos \left(\frac{A_{2}}{1-i r \gamma}\right), \frac{A_{4}}{1-\sqrt{p-1}}\right\} \rightarrow
$$

$$
\begin{gathered}
\left\{\frac{A_{1}}{1-i \sqrt{\gamma}} \sin \left(\frac{A_{2}}{1-i r \gamma}\right), 0, \frac{A_{1}}{1-i \sqrt{\gamma}} \cos \left(\frac{A_{2}}{1-i r \gamma}\right),\left|\frac{A_{4}}{1-\sqrt{p-1}}\right|\right\}, \\
\Pi_{2}:\left\{\frac{A_{1}}{1-i \sqrt{\gamma}} \sin \left(\frac{A_{2}}{1-i r \gamma}\right), 0, \frac{A_{1}}{1-i \sqrt{\gamma}} \cos \left(\frac{A_{2}}{1-i r \gamma}\right),\left|\frac{A_{4}}{1-\sqrt{p-1}}\right|\right\} \rightarrow \\
\left\{\frac{A_{1}}{1-i \sqrt{\gamma}} \sin \left(\frac{A_{2}}{1-i r \gamma}\right), 0, \frac{A_{1}}{1-i \sqrt{\gamma}} \cos \left(\frac{A_{2}}{1-i r \gamma}\right),\left|\frac{\frac{A_{4}}{1-\sqrt{p-1}}}{2}\right|\right\}, \ldots, \\
\Pi_{m}:\left\{\frac{A_{1}}{1-i \sqrt{\gamma}} \sin \left(\frac{A_{2}}{1-i r \gamma}\right), 0, \frac{A_{1}}{1-i \sqrt{\gamma}} \cos \left(\frac{A_{2}}{1-i r \gamma}\right),\left|\frac{A_{4}}{\frac{1-\sqrt{p-1}}{m-1}}\right|\right\} \rightarrow \\
\left\{\frac{A_{1}}{1-i \sqrt{\gamma}} \sin \left(\frac{A_{2}}{1-i r \gamma}\right), 0, \frac{A_{1}}{1-i \sqrt{\gamma}} \cos \left(\frac{A_{2}}{1-i r \gamma}\right),\left|\frac{A_{4}}{1-\sqrt{p-1}}\right|\right\}
\end{gathered}
$$

Then we get

$$
\lim _{m \rightarrow \infty} \Pi_{m}\left\{\frac{A_{1}}{1-i \sqrt{\gamma}} \sin \left(\frac{A_{2}}{1-i r \gamma}\right), 0, \frac{A_{1}}{1-i \sqrt{\gamma}} \cos \left(\frac{A_{2}}{1-i r \gamma}\right), \frac{A_{4}}{1-\sqrt{p-1}}\right\} .
$$

Thus, $-x_{1}^{2}-x_{2}^{2}-x_{3}^{2}+x_{4}^{2}=\left(\frac{A_{1}}{1-i \sqrt{\gamma}}\right)^{2}$, which is the geodesic retraction great circle $S_{2}^{1} \subset S_{2}^{3}$ with $x_{2}=x_{4}=0$. Therefore, $H_{n}\left(S_{1}^{1}\right) \subset S_{2}^{3}$ is isomorphic to $Z$, for $n=0,1$ and $H_{n}\left(S_{1}^{1} \subset S_{2}^{3}\right)=0$, for $n \geq 2$.

Corollary 2: Under the folding $\Pi_{m}\left(x_{1}, x_{2}, x_{3}, x_{4}\right)=$ $\left(x_{1}, x_{2}, x_{3},\left|\frac{x_{4}}{m}\right|\right)$, the homology group of the limit of foldings of the hypersphere $S_{1}^{3}$, be a hypersphere $S_{1}^{2} \subset S_{1}^{3}$ is isomorphic to the identity group or isomorphic to $Z$.

Corollary 3: Under the folding $\Pi_{m}\left(x_{1}, x_{2}, x_{3}, x_{4}\right)=$ $\left(x_{1}, x_{2}, x_{3},\left|\frac{x_{4}}{m}\right|\right)$ and $A_{3}=0$, the homology group of the limit of foldings of the a hypersphere $S_{1}^{3} \subset \beta^{4}$, is a great circle $S_{3}^{1} \subset S_{1}^{3}$, which is either identity group or isomorphic to $Z$.

CorollarY 4: Under the folding $\Pi_{m}\left(x_{1}, x_{2}, x_{3}, x_{4}\right)=$ $\left(x_{1}, x_{2}, x_{3},\left|\frac{x_{4}}{m}\right|\right)$ and $A_{3}=0$, the fundamental group of the deformation retract of $S_{1}^{3}$ onto the great circle $S_{3}^{1} \subset S_{1}^{3}$ is either identity group or isomorphic to $Z$.

Theorem 4: Under the folding $\Pi_{m}\left(x_{1}, x_{2}, x_{3}, x_{4}\right)=$ $\left(\left|\frac{x_{1}}{m}\right|,\left|\frac{x_{2}}{m}\right|, x_{3}, x_{4}\right)$ the homology group of the limit foldings of the Buchdahi space $\beta^{4}$ is either identity group or isomorphic to $Z$. 
Proof: Now consider the Buchdahi space $\beta^{4}$ of dimension four and let $\prod_{m}: \beta^{4} \rightarrow \beta^{4}$ be given by $\Pi_{m}\left(x_{1}, x_{2}, x_{3}, x_{4}\right)=\left(\left|\frac{x_{1}}{m}\right|,\left|\frac{x_{2}}{m}\right|, x_{3}, x_{4}\right)$. Then, the isometric chain folding of the Buchdahi space $\beta^{4}$ into itself may be defined by

$$
\begin{aligned}
& \Pi_{1}:\left\{\frac{A_{1}}{1-i \sqrt{\gamma}} \sin \left(\frac{A_{2}}{1-i r \gamma}\right), \frac{A_{1}}{1-i \sqrt{\gamma}} \sin \left(\frac{A_{2}}{1-i r \gamma}\right) \sin \left(\frac{A_{3}}{1-i r \gamma \sin (\varphi)}\right),\right. \\
& \left.\frac{A_{1}}{1-i \sqrt{\gamma}} \cos \left(\frac{A_{2}}{1-i r \gamma}\right), \frac{A_{4}}{1-\sqrt{p-1}}\right\} \rightarrow\left\{\left|\frac{A_{1}}{1-i \sqrt{\gamma}} \sin \left(\frac{A_{2}}{1-i r \gamma}\right)\right|,\right. \\
& \left.\left|\frac{A_{1}}{1-i \sqrt{\gamma}} \sin \left(\frac{A_{2}}{1-i r \gamma}\right) \sin \left(\frac{A_{3}}{1-i r \gamma \sin (\varphi)}\right)\right|, \frac{A_{1}}{1-i \sqrt{\gamma}} \cos \left(\frac{A_{2}}{1-i r \gamma}\right), \frac{A_{4}}{1-\sqrt{p-1}}\right\}, \\
& \Pi_{2}:\left\{\left|\frac{A_{1}}{1-i \sqrt{\gamma}} \sin \left(\frac{A_{2}}{1-i r \gamma}\right)\right|,\left|\frac{A_{1}}{1-i \sqrt{\gamma}} \sin \left(\frac{A_{2}}{1-i r \gamma}\right) \sin \left(\frac{A_{3}}{1-i r \gamma \sin (\varphi)}\right)\right|,\right. \\
& \left.\frac{A_{1}}{1-i \sqrt{\gamma}} \sin \left(\frac{A_{2}}{1-i r \gamma}\right) \frac{A_{4}}{1-\sqrt{p-1}}\right\} \rightarrow\left\{\frac{\frac{A_{1}}{1-i \sqrt{\gamma}} \sin \left(\frac{A_{2}}{1-i r \gamma}\right)}{2} \mid,\right. \\
& \left.\left|\frac{\frac{A_{1}}{1-i \sqrt{\gamma}} \sin \left(\frac{A_{2}}{1-i r \gamma}\right) \sin \left(\frac{A_{3}}{1-i r \gamma \sin (\varphi)}\right)}{2}\right|, \frac{A_{1}}{1-i \sqrt{\gamma}} \cos \left(\frac{A_{2}}{1-i r \gamma}\right), \frac{A_{4}}{1-\sqrt{p-1}}\right\}, \ldots, \\
& \Pi_{m}:\left\{\left|\frac{\frac{A_{1}}{1-i \sqrt{\gamma}} \sin \left(\frac{A_{2}}{1-i r \gamma}\right)}{m-1}\right|,\left|\frac{\frac{A_{1}}{1-i \sqrt{\gamma}} \sin \left(\frac{A_{2}}{1-i r \gamma}\right) \sin \left(\frac{A_{3}}{1-i r \gamma \sin (\varphi)}\right)}{m-1}\right|,\right. \\
& \left.\frac{A_{1}}{1-i \sqrt{\gamma}} \cos \left(\frac{A_{2}}{1-i r \gamma}\right), \frac{A_{4}}{1-\sqrt{p-1}}\right\} \rightarrow\left\{\left|\frac{\frac{A_{1}}{1-i \sqrt{\gamma}} \sin \left(\frac{A_{2}}{1-i r \gamma}\right)}{m}\right|\right. \\
& \left.\left|\frac{\frac{A_{1}}{1-i \sqrt{\gamma}} \sin \left(\frac{A_{2}}{1-i r \gamma}\right) \sin \left(\frac{A_{3}}{1-i r \gamma \sin (\varphi)}\right)}{m}\right|, \frac{A_{1}}{1-i \sqrt{\gamma}} \cos \left(\frac{A_{2}}{1-i r \gamma}\right), \frac{A_{4}}{1-\sqrt{p-1}}\right\}
\end{aligned}
$$

Then we get

$$
\lim _{m \rightarrow \infty} \prod_{m}\left\{0,0, \frac{A_{1}}{1-i \sqrt{\gamma}} \cos \left(\frac{A_{2}}{1-i r \gamma}\right), \frac{A_{4}}{1-\sqrt{p-1}}\right\} .
$$

Thus, $\quad-x_{1}^{2}-x_{2}^{2}-x_{3}^{2}+x_{4}^{2}=\left(\frac{A_{1}}{1-i \sqrt{\gamma}} \cos \left(\frac{A_{2}}{1-i r \gamma}\right)\right)^{2}+\left(\frac{A_{4}}{1-\sqrt{p-1}}\right)^{2}$, which is hypersurface $\beta^{1} \subset \beta^{4}$ with $x_{1}=x_{2}=0$. Therefore $H_{n}\left\{\beta^{1}-\left(\delta_{i}\right)\right\} \subset\left\{\beta^{4}-\left(\delta_{i}\right)\right\}$ is isomorphic to $Z$, for $n=0,1$ and $H_{n}\left\{\beta^{1}-\left(\delta_{i}\right)\right\} \subset\left\{\beta^{4}-\left(\delta_{i}\right)\right\}$ is the identity, for $\mathrm{n} \geq 2$.

Theorem 5: Under the folding $\Pi_{m}\left(x_{1}, x_{2}, x_{3}, x_{4}\right)=$ $\left(\left|\frac{x_{1}}{m}\right|,\left|\frac{x_{2}}{m}\right|,\left|\frac{x_{3}}{m}\right|,\left|\frac{x_{4}}{m}\right|\right)$, the homology group of the limit of foldings of the Buchdahi space $\beta^{4}$ is either identity group or isomorphic to $Z$.
Proof: Consider the four dimension Buchdahi space $\beta^{4}$ and let $\prod_{m}: \beta^{4} \rightarrow \beta^{4}$ be given by $\Pi_{m}\left(x_{1}, x_{2}, x_{3}\right.$, $\left.x_{4}\right)=\left(\left|\frac{x_{1}}{m}\right|,\left|\frac{x_{2}}{m}\right|,\left|\frac{x_{3}}{m}\right|,\left|\frac{x_{4}}{m}\right|\right)$. Then, the isometric chain folding of the Buchdahi space $\beta^{4}$ into itself get $\lim _{m \rightarrow \infty} \prod_{m}\{0,0,0,0\}$, which a zero- dimensional Buchdahi space. Thus, it is a point and the homology group of a point is the identity group or isomorphic to $Z$.

Corollary 5: The homology group of the end limits of foldings of the $\mathrm{n}$-dimensional manifold $F^{n}$ homeomorphic to $\mathrm{n}$-dimensional Buchdahi space $\beta^{n}$ into itself is the identity group or isomorphic to $Z$.

Theorem 6: The homology group of the minimal retraction of the n-dimensional manifold $F^{n}$ homeomorphic to $\mathrm{n}$-dimensional Buchdahi space $\beta^{n}$ is either identity group or isomorphic to $Z$.

Proof: Let $r_{i}:\left\{F^{n}-\left(\beta_{i}^{n}\right)\right\} \rightarrow F^{n-1}$ be the retraction map. Then, we have the following chains

$$
\begin{aligned}
& \left\{F^{n}-\left(\beta_{i}^{n}\right)\right\} \stackrel{r_{1}^{1}}{\longrightarrow}\left\{F_{1}^{n}-\left(\beta_{1}^{n}\right)\right\} \stackrel{r_{2}^{1}}{\longrightarrow}\left\{F_{2}^{n}-\left(\beta_{2}^{n}\right)\right\} \rightarrow \ldots \\
& \left\{F_{2}^{n}-\left(\beta_{2}^{n}\right)\right\} \stackrel{\lim _{i}^{n_{i}^{1}} \longrightarrow}{\longrightarrow} F^{n-1} \\
& \left\{F^{n-1}-\left(\beta_{i}^{n-1}\right)\right\} \stackrel{r_{1}^{2}}{\longrightarrow}\left\{F_{1}^{n-1}-\left(\beta_{1}^{n-1}\right)\right\} \stackrel{r_{2}^{2}}{\longrightarrow}\left\{F_{2}^{n-1}-\left(\beta_{2}^{n}\right)\right\} \rightarrow \ldots \\
& \left\{F_{n-1}^{n-1}-\left(\beta_{n-1}^{n-1}\right)\right\} \stackrel{\lim _{i}^{r_{i}^{2}} \longrightarrow}{\longrightarrow} F^{n-2}, \ldots, \\
& \left\{F^{1}-\left(\beta_{i}^{1}\right)\right\} \stackrel{r_{1}^{n}}{\longrightarrow}\left\{F_{1}^{1}-\left(\beta_{1}^{1}\right)\right\} \stackrel{r_{2}^{n}}{\longrightarrow}\left\{F_{2}^{1}-\left(\beta_{2}^{1}\right)\right\} \rightarrow \ldots \\
& \left\{F_{n-1}^{1}-\left(\beta_{n-1}^{1}\right)\right\} \stackrel{\lim _{i \rightarrow \infty}^{r_{i}^{n}} \longrightarrow}{\longrightarrow} F^{0} .
\end{aligned}
$$

Thus from the above chain the minimal retractions of the n-dimensional manifold $F^{n}$ coincides with the zero-dimensional space which is the limit of retractions. Thus, it is a point and the homology group of a point is either identity group or isomorphic to $Z$.

Theorem 7: The homology group of the retraction of Buchdahi plane $\beta^{2}$ is either identity group or isomorphic to $Z$.

Proof: Since $\gamma=\frac{1}{2} \ln B C[6]$, if $B=C$, then $\gamma=\ln B C$. When $\ln B=1$, implies $\gamma=1$. Hence (1) becomes $d s^{2}=\left(-d r^{2}+r^{2} d \theta^{2}\right.$ $\left.+r^{2} \sin ^{2} \theta d \phi^{2}\right)+p^{-1} d t^{2}$. Also, under the condition $t=\phi=0$, then $d s^{2}$ implies that $d s^{2}=-d r^{2}-r^{2} d \theta^{2}$. Now $S_{\sigma}^{1}$ is a retract of $\beta^{2}-\{(0,0)\}$, where $r: \beta^{2}-\{(0,0)\} \rightarrow S_{\sigma}^{1}$ defined 
by $r(x)=\frac{x}{\|x\|}$ is a continuous map. Therefore $H_{n}\left(S_{\sigma}^{1}\right)$ is either identity group or isomorphic to $Z$.

THEOREM 8: The homology group of the deformation retract of the Buchdahi plane $\beta^{2}$ is either identity group or isomorphic to $Z$.

Proof: Since $S_{\sigma}^{1}$ is a retract of $\beta^{2}-\{(0,0)\}$, but a subset A of a Buchdahi plane $\beta^{2}$ is said to be a deformation retract of the Buchdahi plane $\beta^{2}$ if there exists a homotopy map $F:\left(\beta^{2}-\{(0,0)\}\right) \times[0,1] \rightarrow\left(\beta^{2}-\{(0,0)\}\right)$ defined as $F(x, t)=(1-t) x+t\left(\frac{x}{\|x\|}\right)$ such that $F_{0}=i d_{B}$ and $F_{1}:\left(\beta^{2}-\{(0,0)\}\right) \rightarrow\left(\beta^{2}-\{(0,0)\}\right)$ satisfies $F_{1}(x) \in A$ for every $x \in\left(\beta^{2}-\{(0,0)\}\right)$ and $F_{1}\left(S_{\sigma}^{1}\right)=S_{\sigma}^{1}$ for every $S_{\sigma}^{1} \in A$. Hence $\mathrm{F}$ a deformation retract of the Buchdahi plane onto $S_{\sigma}^{1}$ and $H_{n}\left(S_{\sigma}^{1}\right)$ is either identity group or isomorphic to $Z$.

THEOREM 9: The homology group of any types of folding of $S^{n} \subset \beta^{n+1}$ such that $\operatorname{dim} \mathfrak{I}\left(S^{n}\right)=\operatorname{dim}\left(S^{n}\right), n \geq 2$, is either identity group or isomorphic to $Z$.

Proof: Now, consider the foldings with singularity of $S^{n} \subset \beta^{n+1}$ to a subset of $S^{n} \subset \beta^{n+1}$ such that $\operatorname{dim} \mathfrak{I}\left(S^{n}\right)=\operatorname{dim}\left(S^{n}\right)$, then all loops of $\mathfrak{I}\left(S^{n}\right)$ are homotopic to the identity loop, and hence the fundamental group of this types of folding is the identity group as in Figure 1. Again, consider the foldings without singularity of $S^{n} \subset \beta^{n+1}$ and also the homology group of this types of foldings is either identity group or isomorphic to $Z$ as in Figure 2 .

THEOREM 10: Let $M \subset \beta^{2}$ be the union of the circles $C^{n}=\underset{i \in Z-\{0\}}{\bigcup} S^{1}\left(\left(\frac{1}{i}, 0\right), \frac{1}{i}\right) \subset \beta^{2}$. Then there are folding $F: M \rightarrow M$ and retractions $r: M \rightarrow C^{n}$ such that $H_{1}\left(F_{m}(M)\right)=H_{1}\left(r_{m}(M)\right), H_{1}\left(F_{m}(M)\right)$ is either identity group or isomorphic to $Z$.

Proof: Let $F_{n}: M \rightarrow M$ be a folding such that $F_{n}\left(C^{n}\right)=C$,

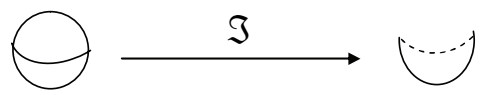

Figure 1. Folding with singularity of the loop.

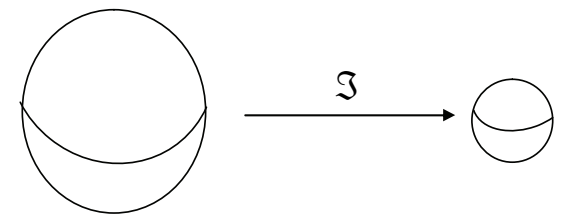

Figure 2. Folding without singularity of hypersphere . then $F_{n}(M)=C^{n}$. Also, consider the retraction $r_{n}: M \rightarrow C^{\prime \prime}$, which collapsing all $C_{j}$ except $C^{n}$ to the origin and too $r_{n}: M=C^{n}$, then $H_{1}\left(F_{m}(M)\right)=H_{1}\left(r_{m}(M)\right)$. Now, if $n \rightarrow \infty$ then $C^{n}$ is a point and so $H_{1}\left(F_{m}(M)\right)=Z$ otherwise if $n \nrightarrow \infty$ then $C^{n}$ is a circle and $H_{1}\left(F_{m}(M)\right)$ is either identity group or isomorphic to $Z$. See Figure 3.

TheOREM 11: Let $M \subset \beta^{2}$ be the union of the circles $C^{n}=\underset{i \in Z-\{0\}}{\cup} S^{1}\left(\left(\frac{1}{i}, 0\right), \frac{1}{i}\right) \subset \beta^{2}$. Then there are folding $F: M \rightarrow M$ which induces a folding $\bar{F}: H_{1}(M) \rightarrow H_{1}(M)$ such that $\bar{F}\left(H_{1}(M)\right)=H_{1}(F(M))$ and $\bar{F}\left(H_{1}(M)\right)$ is uncountable.

Proof: Let $F_{n}: M \rightarrow M$ be a folding such that $F_{n}\left(C^{n}\right)=F_{n}\left(\underset{i \in Z-\{0\}}{\cup} S^{1}\left(\left(\frac{1}{i}, 0\right), \frac{1}{i}\right) \subset \beta^{2}\right)=C^{n}$. Also, $F_{n} \overline{\left(C_{1}^{n}\right)}=F_{n}$ $\left(\underset{i \in Z-\{0\}}{\bigcup} S^{1}\left(\left(\frac{1}{i}, 0\right), \frac{1}{i}\right) \subset \beta^{2}\right)=C^{n}$ i.e. $F_{n}\left(C_{i}^{n}\right)=F_{n} \overline{\left(C_{i}^{n}\right)}=\underset{i \in Z-\{0\}}{\bigcup}$ $S^{1}\left(\left(\frac{1}{i}, 0\right), \frac{1}{i}\right) \subset \beta^{2}$. Thus we get the induced folding $\bar{F}: H_{1}(M) \rightarrow H_{1}(M)$ such that $\bar{F}\left(H_{1}(M)\right)=H_{1}(F(M))$ since $H_{1}(F(M))$ is uncountable, it follows that $\bar{F}\left(H_{1}(M)\right)$ is uncountable. See Figure 4.

Corollary 6: Let $M \subset \beta^{2}$ be the union of the circles $C^{n}=\underset{i \in Z-\{0\}}{\cup} S^{1}\left(\left(\frac{1}{i}, 0\right), \frac{1}{i}\right) \subset \beta^{2}$. If $\mathfrak{I}: M \rightarrow M$ be a folding defined as $\mathfrak{I}(x, y)=(|x|,|y|)$. Then $H_{1}(\mathfrak{I}(M))=0$.

\section{Conclusion}

In this paper we achieved the approval of the important of the homology group in the submanifolds of Buchdahi Space by using some geometrical transformations. The relations

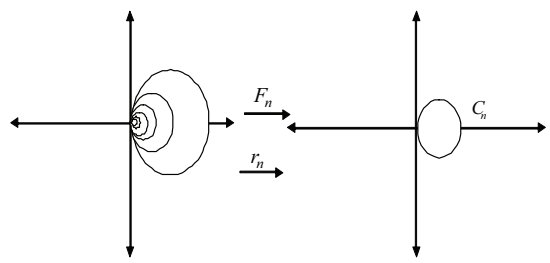

Figure 3. Conditional retractions of union of circles.

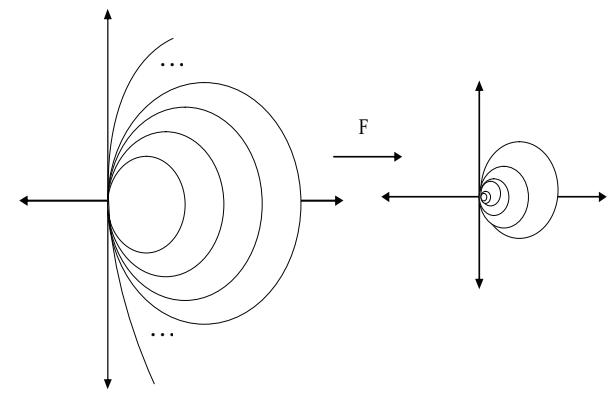

Figure 4. Conditional foldings of union of circles. 
between folding, retractions, deformation retract, limits of folding and limits of retractions of the homology group in the submanifolds of Buchdahi Space are discussed. The connection between limits of the folding and the homology group are obtained. New types of minimal retractions on the homology group are deduced.

\section{References}

1. El-Ahmady A E (2007). The variation of the density on chaotic spheres in chaotic space-like Minkowski space time, Chaos, Solitons and Fractals, vol 31(5), 1272-1278.

2. El-Ahmady A E (2007). Folding of fuzzy hypertori and their retractions, Proc. Math. Phys. Soc., vol 85, No. 1, 1-10.

3. El-Ahmady A E (2006). Limits of fuzzy retractions of fuzzy hyperspheres and their foldings, Tamkang Journal of Mathematics, vol 37, No. 1, 47-55.

4. El-Ahmady A E (2004). Fuzzy folding of fuzzy horocycle, Circolo Matematico di Palermo Serie II, Tomo L III, 443450.

5. El-Ahmady A E (2004). Fuzzy Lobachevskian space and its folding, The Journal of Fuzzy Mathematics, vol 12, No. 2, 609-614.

6. El-Ahmady A E (1994). The deformation retract and topological folding of Buchdahi space, Periodica Mathematica Hungarica, vol 28, 19-30.

7. El-Ahmady A E (2011). The geodesic deformation retract of Klein bottle and its folding, The International Journal of Nonlinear Science, vol 9, No. 3, 1-8.

8. El-Ahmady A E (2013). Folding and fundamental groups of Buchdahi space, Indian Journal of Science and Technology, vol 6, No. 1, 3940-3945.

9. El-Ahmady A E (2012). Retraction of null helix in Minkowski 3-space, The Scientific Journal of Applied Research, vol 1(2), 28-33.

10. El-Ahmady A E (2013). On elastic Klein bottle and fundamental groups, Applied Mathematics, vol 4, No. 3, 499-504.

11. El-Ahmady A E (2011). Retraction of chaotic black hole, The Journal of Fuzzy Mathematics, vol 19, No. 4, 833-838.

12. El-Ahmady A E (2013). On the fundamental group and folding of Klein bottle, International Journal of Applied Mathematics and Statistics, vol 37, No. 6, 56-64.

13. El-Ahmady A E (2013). Fuzzy elastic Klein bottle and its retractions, International Journal of Applied Mathematics and Statistics, vol 42, No. 12, 94-102.

14. El-Ahmady A E (2013). Folding and fundamental groups of flat Robertson-Walker Space, Indian Journal of Science and Technology, vol 6, No. 4, 4235-4242.

15. El-Ahmady A E (2013). Fundamental groups and folding of Minkowski space, European Journal of Scientific Research, vol 104, No. 2, 284-293.
16. El-Ahmady A E (2013). Retraction of chaotic Schwarzchild space, European Journal of Scientific Research, vol 104, No. 3, 333-339.

17. El-Ahmady A E (2013). The isonormal folding of fuzzy manifolds and its retractions, European Journal of Scientific Research, vol 104, No. 3, 340-347.

18. El-Ahmady A E, and Al-Rdade A (2013). Deformation retracts of the Reissner-Nordström spacetime and its foldings, American Journal of Applied Sciences, vol 10, No. 7, 740-745.

19. El-Ahmady A E, and Al-Rdade A (2013). On the geometry of fuzzy Reissner-Nordström spacetime and its foldings, European Journal of Scientific Research, vol 104, No. 2, 294303.

20. El-Ahmady A E, and Al-Rdade A (2013). Fuzzy retraction of fuzzy spacetime, Indian Journal of Science and Technology, vol 6, No. 6, 4687-4696.

21. El-Ahmady A E, and Al-Hazmi N (2013). On the folding of Buchdahi space, European Journal of Scientific Research, vol 100, No. 2, 315-322.

22. El-Ahmady A E, and Al-Hazmi N (2013). The exponential deformation retract of the hypertori and its foldings, European Journal of Scientific Research, vol 104, No. 4, 561568.

23. El-Ahmady A E, and Al-Onema K (2013). The deformation retractions of fuzzy sphere in fuzzy Lobachevsky space and its folding, European Journal of Scientific Research, vol 104, No. 4, 549-560.

24. El-Ahmady A E (2012). Folding and unfolding of chaotic spheres in chaotic space-like Minkowski space-time, The Scientific Journal of Applied Research, vol 1(2), 34-43.

25. Abu-Saleem M (2013). Homology group on the dynamical trefoil knot, Indian Journal of Science and Technology, vol 6 (5), 4514-4518.

26. Abu-Saleem M (2013). On chaotic Cartesian product of graphs and their retractions, International Journal of Nonlinear Science, vol 15, No. 1, 86-90.

27. Abu-Saleem M (2013). On chaotic homotopy group, Advanced Studies in Contemporary Mathematics, vol 23, No. $1,69-75$.

28. Abu-Saleem M (2012). On the dynamical hyperbolic 3-spaces and their deformation retracts, Proceedings of the Jangjeon Mathematical Society, vol 15, No. 2, 189-193.

29. Abu-Saleem M (2011). Dynamical chaotic homotopy group and its applications, International Journal of Nonlinear Science, vol 11, No. 2, 206-212.

30. Abu-Saleem M (2011). On dynamical chaotic de sitter spaces and their deformation retracts, Proceedings of the Jangjeon Mathematical Society, vol 14, 231-238.

31. Abu-Saleem M (2011). On dynamical chaotic Weyle representations of the vacuum $\mathrm{C}$ metric and their retractions, International Journal of Mathematical Combinatorics, vol 3, 47-54. 
32. M. Abu-Saleem (2010). Folding on the wedge sum of graphs and their fundamental group, Applied Sciences, vol 12, (14-19), 14-19.

33. Abu-Saleem M (2010). Homology group on manifolds and their foldings, Tamkang Journal of Mathematics, vol 41, No. $1,31-38$.

34. Abu-Saleem M (2010). Folding and unfolding of manifolds and their fundamental groups, International Journal of Contemporary Mathematical Sciences, vol 5, No.1, 1-19.

35. Abu-Saleem M (2010). Dynamical knot and their fundamental group, International Journal of Mathematical Combinatorics, vol 1, 80-86.

36. Abu-Saleem M (2010). Dynamical manifolds and their fundamental group, Advanced Studies in Contemporary Mathematics, vol 20, No.1, 125-131.
37. Abu-Saleem M (2010). Conditional fractional folding of a manifold and their fundamental group, Advanced Studies in Contemporary Mathematics, vol 20, No. 2, 271-277.

38. Abu-Saleem M (2008). Folding on the chaotic Cartesian product of manifolds and their fundamental group, Tamkang Journal of Mathematics, vol 39, No. 4, 353-361.

39. Lefeschets S (1942). Algebraic Topology, American Mathematical Society.

40. Naber G L (2011). Topology, Geometry and Gauge Fields, Foundations, Chapter 1, Springer-Verlage New York, Berlin.

41. Arkowitz M (2011). Introduction to Homotopy Theory, Chapter 1, Springer- Verlage.

42. Strom J (2011). Modern classical homotopy theory, Chapter 1 and 13, American Mathematical Society. 